\title{
Building Campus Sports Information Learning Platform Based on Intelligent Sensor Network
}

\author{
Chao Chen, ${ }^{1}$ Zhiming Wu, ${ }^{2}$ Qing Chen $\mathbb{D}^{3},{ }^{3}$ and Xinwen $\mathrm{Li}^{4}$ \\ ${ }^{1}$ College of Physical Education, Jilin Normal University, Siping 136000, Jilin, China \\ ${ }^{2}$ State Grid Yiwu Power Supply Company, Yiwu 322000, Zhejiang, China \\ ${ }^{3}$ College of Physical Education, Sichuan University, Chengdu 610065, Sichuan, China \\ ${ }^{4}$ Department of Physical Education, University of Electronic Science and Technology of China, Chengdu 610054, Sichuan, China \\ Correspondence should be addressed to Qing Chen; chen_qing@scu.edu.cn
}

Received 7 December 2021; Revised 25 December 2021; Accepted 17 January 2022; Published 10 February 2022

Academic Editor: Deepak Kumar Jain

Copyright $\odot 2022$ Chao Chen et al. This is an open access article distributed under the Creative Commons Attribution License, which permits unrestricted use, distribution, and reproduction in any medium, provided the original work is properly cited.

\begin{abstract}
In the present era of rapid network growth and in the face of growing national power rivalry, education, as the cornerstone of a nation and a national prosperity symbol, has been considered by countries around the world as an important indicator of comprehensive national power. From this perspective, education should keep pace with the times and integrate into the Internet era of "Internet+" and big data. Education computerization is a great product of education's absorption into the Internet era and is an important content and an important symbol of education modernization. It can realize the modernization of education, and its construction can better reduce the teaching differences between regions, improve teaching quality, and balance resources in education. Moreover, it is conducive to the implementation of quality education and the cultivation of innovative talents, and it is an effective way to realize a learning society and build a lifelong education system. The experiment draws the results of the experiment by comparing the results of students receiving two different education methods. From the specific performance point of view, the average long jump score of boys in the experimental group was $4.40 \mathrm{~m}$, while the average score of boys in the control group was $4.28 \mathrm{~m}$, and the score of the experimental group was $0.12 \mathrm{~m}$ higher than that of the control group. The average long jump performance of the girls in the experimental group was $3.52 \mathrm{~m}$, and the average performance of the girls in the control group was $3.30 \mathrm{~m}$. In the long jump scores assessed in the second grade, the full score of boys is $4.35 \mathrm{~m}$, and the score of girls is $3.49 \mathrm{~m}$; the average scores of the experimental group students reached full marks. Compared with traditional teaching methods, informationbased teaching facilitates the analysis of key and difficult points for teachers, greatly improves the quality and efficiency of teaching, and improves the teaching level of the classroom.
\end{abstract}

\section{Introduction}

Wireless sensor networks include the logical world of information and the physical world of things, which will change the way people interact with the physical world and expand people's ability to understand the physical world. However, with the increasing complexity of the environment, the simple data collection field achieved by traditional methods can no longer meet the needs of users. People urgently need rich and comprehensive environmental data (images, audio, video) to complete detailed and accurate environmental data. This monitoring has established a wireless sensor network.
For intelligent sensor networks and sports information teaching, experts at home and abroad have done many researches. Fadi believes that the information-centric sensor network is a model of the wireless sensor network, which focuses on transmitting information from the network according to user needs, rather than acting as a point-topoint data communication network. Introducing learning into such a network can help dynamically identify good data transmission paths by correlating past actions and results, make intelligent adaptations to improve the life of the network, and improve the quality of information delivered by the network to users [1]. Gong et al. explore the optimization of the intelligently optimized remote sports 
teaching system, optimize the system, including system indexes, logical databases, and query structures, and explore system functions. The optimized intelligent physical education system has better functions [2]. Borawake-Satao and Prasad believe that wireless sensor network (WMSN) is expected to become a key technology for future networks. Multimodal information and the low-cost availability of camera sensor nodes are promoting the widespread use of audio, image, and video in various real-time implementations [3]. Tang et al. believe that energy harvesting technology has recently been integrated into wireless sensor networks to improve the energy limitation of nodes. In theory, wireless sensor nodes equipped with energy harvesting modules can work permanently until hardware failure occurs. However, due to power changes, traditional hierarchical network routing protocols cannot be effectively used in energy harvesting wireless sensor networks [4]. Ling et al. constructed a multi-information teaching model for the mobile reading habits and information-based learning needs of modern college students, discussed the practical effects of the multi-information teaching model of inorganic chemistry, and found that this teaching model can make up for the shortcomings of traditional teaching [5]. Sohn et al. believe that emerging applications in the fields of automation, medical imaging, traffic monitoring, and surveillance require real-time data transmission through wireless sensor networks (WSN). The rapid popularization of smart devices and the standardization of machine type communication (MTC) in the next-generation 5G wireless network have added new dimensions to these challenges [6].

From the perspective of theoretical value, it has greatly enriched the theoretical knowledge of physical education classroom teaching, has made a perfect supplement to the traditional physical education teaching theories, explained some fuzzy concepts, and optimized the teaching design theory. The practice of multimedia teaching has a significant impact on physical education. It has greatly improved the level of teaching effectiveness and perfected the current education system. From the perspective of practical value, it uses theory to guide the implementation and integration of teaching-the in-depth practice of classroom teaching. Through teaching, it helps to enhance the relationship between teachers, students, and classmates, promotes students' teamwork ability, helps enhance students' subjective initiative in learning, and further cultivates students' interest in learning.

\section{Intelligent Sensor Network and Informatization Teaching Methods}

The construction of modern distance education resources includes media material library, question library, case library, courseware library, and network course construction, as well as the research and development of teaching support system suitable for multiple teaching modes and modern distance education management system [7]. These contents and their relationships constitute the modern distance education resource system structure, which is directly placed on the relevant equipment or environment so that it can perform independently assigned tasks in a seamless environment [8]. Compared with traditional WSNs and simple environmental data collection tasks, WMSNs can collect information such as audio, video, and photos and have rich and accurate information and accurate data monitoring; the wireless multimedia sensor network is composed of a large number of random deployments or deterministic deployments in the monitoring area [9]. It is small in size, cheap, and low in energy consumption, supports short-distance communication of sensor nodes, and forms a multihop wireless communication network through self-configuration [10]. The structure diagram of the wireless sensor network is shown in Figure 1 [11].

Classification of path monitoring capability issues in sensor networks.

The wireless sensor network serves the material world $[12,13]$, and the difference of the material world directly determines the very different network structure and characteristics. In order to enable the sensor network to complete the access to the target domain information, it is necessary to classify the network path monitoring capabilities, thereby enhancing the deployment monitoring coverage and significance of the monitoring of the sensor network [14].

Classification by perception model

The establishment of the perception model is a prerequisite for the research on the path monitoring capability of the entire wireless sensor network, which affects the coverage performance of the entire network. There are three main perception models for sensor networks.

\section{Boolean perception model}

The sensing range of a node is a circular area with the node as the center and the radius $r$ as the sensing distance (determined by the hardware characteristics of the node) [15], as shown in Figure 2. On the other hand, each node can only detect the environment and track targets within its sensor range. Under the Boolean perception model, the monitoring area is divided into two parts: coverage area and vacant area. If a location is perceived by at least one node, it is called a coverage area; otherwise, it is called a vacant area.

2.1. General Perception Model. The above-mentioned Boolean perception model simplifies the coverage problem and facilitates an in-depth study of the problem. The disadvantage is that as the distance between the sensor and the measuring point increases, the sensor's perception ability is decreasing. But the Boolean perception model cannot obtain this information, and the general perception model solves this problem well [16], as shown in Figure 3. For a node $f$, the induced signal at any point $q$ is as follows:

$$
F(f, q)=\frac{a}{\left\{\begin{array}{l}
d(f, q)^{b} \\
0
\end{array}\right.} A \leq d(f, q) \leq B .
$$




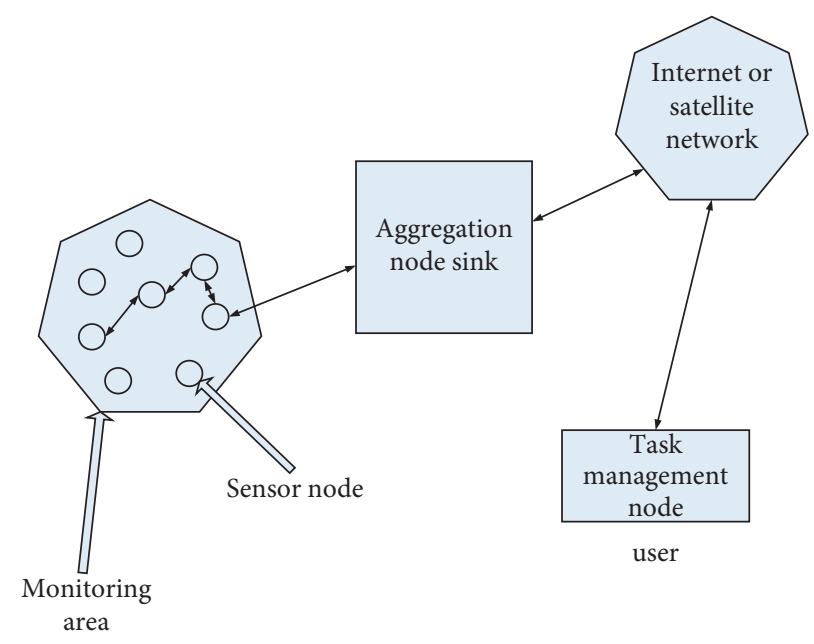

FIgURE 1: Wireless sensor network.

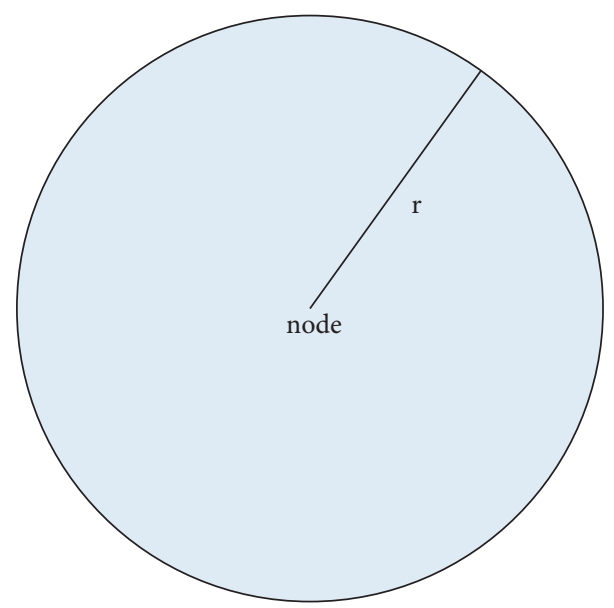

FIGURE 2: Boolean perception model.

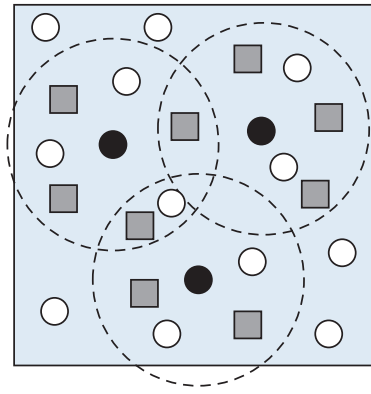

(a)

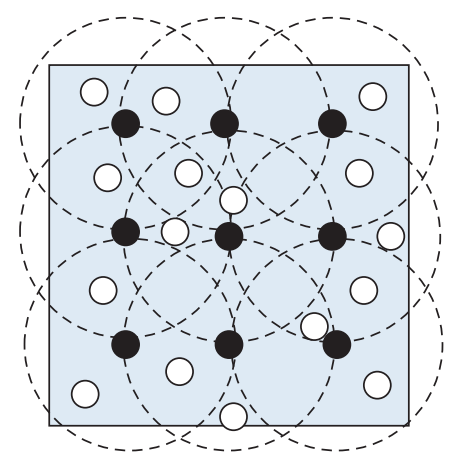

(b)

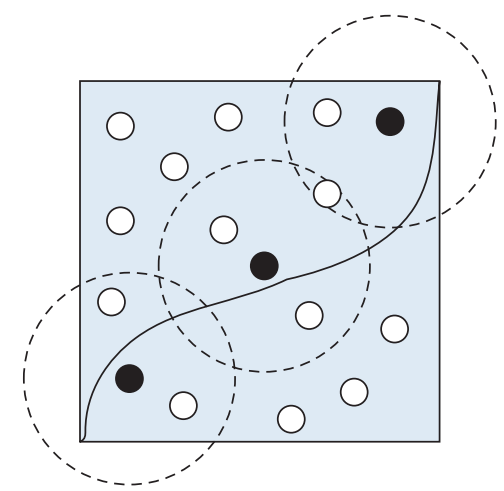

(c)

Figure 3: (a) Point coverage. (b) Plane coverage. (c) Barrier coverage.

Among them, $F(f, q)$ is the Euclidean distance between sensor $f$ and point $q, a$ and $b$ are parameters related to technology and environment, and $A$ and $B$ represent the range of node perception capabilities.
2.1.1. Classified by Coverage Area. According to different applications, the coverage area of wireless sensor networks can be divided into three categories: point coverage, area coverage, and fence coverage, as shown in Figure 3. 
(a) Point coverage

In the application of wireless sensor networks, there are some nodes that have an important impact on the global environment. These points need to be studied. Some key points for monitoring and analysis are called point coverage.

(b) Area coverage

In many applications of wireless sensor networks, it is generally difficult to determine the location of the event point in the area, such as the trigger point of a forest fire and the specific location of enemy personnel on the battlefield. At this time, the entire area needs to be monitored, which is called area coverage.

(c) Fence coverage

Wireless sensor networks are often used in battlefield monitoring applications, such as monitoring enemy targets crossing the monitoring area. By analyzing the weak link in the monitoring area (that is, the place where the enemy is most likely to traverse), we select the appropriate node to monitor the target's traversal path, which is called fence coverage.

2.1.2. Wireless Sensor Network Path Monitoring Capability Based on Detection Coverage. Based on the straight-line strategy, the article starts with the node deployment method and analyzes that the nodes satisfy the Poisson distribution. When the target moves from point $s$ to point $d$, it adopts a straight-line approach, and the area of the tube-shaped area is minimized. At this time, the probability of being monitored is the smallest, and then, the path monitoring ability under delay perception and no delay perception is analyzed [17].

2.1.3. Straight-Line Strategy. Random coverage is generally used in dangerous places such as battlefields or other places with harsh environments, and nodes are randomly and evenly scattered in the target area. This article studies the deployment of nodes under random coverage. The nodes are generally scattered evenly in the monitoring area by aircraft to meet the Poisson distribution.

$$
Q[M(Y)=k]=e^{-\lambda\left\|Z_{Y}\right\|}-\frac{\left(\lambda\left\|Z_{Y}\right\|\right) k}{k !} k \in M_{0},
$$

where $Y$ represents the target area, $k$ represents the number of sensor nodes monitored at any point in the area, $\left\|Z_{Y}\right\|$ represents the area sensed by a node, and $\lambda$ represents the node density. In the wireless sensor network, the sensor's ability to monitor the target depends on the target's understanding of the network. In the case of detection coverage, the target does not know the location of the node and randomly selects a path as the traversal path. The picture depicts the situation where the target moves from point $f$ to point $d$ along the path $p$. The tube-shaped area enclosed by two curves (p1 and p2) with a distance of $r$ along the path $p$ is defined as the monitoring area of path $p$ and is denoted as $Z_{p}$. At this time, the target moving along the path $p$ will be monitored by the nodes in $Z_{p}$. The area of $Z_{p}$ is recorded as $Z_{p}$, and the number of nodes in $Z_{p}$ is recorded as $M_{p}$. Therefore, the probability that a target along path $p$ will be detected by at least one node is

$$
Q_{b}(q)=1-q\left(M_{q}=0\right)=1-e^{-\lambda\|Z q\|} .
$$

It can be seen from formula (2) that in order to minimize the probability of being monitored, the target should choose a path with the lowest monitoring domain. For all possible paths between $S$ and $D$, the monitoring area generated by the linear trajectory is the smallest, as shown in Figure 4. Therefore, in order to minimize the probability of the target being monitored, the best strategy is to take a direct path. Denote the length of the line SD as $x$; then $\left\|Z_{p}\right\|=2 r x+\pi r^{2}$. The probability of the target being monitored along the path $\mathrm{SD}$ is

$$
Q_{d}(x)=1-e^{-\lambda\left(2 r x+\pi r^{2}\right)}
$$

\subsection{Path Monitoring Capabilities under the Delay Perception} Model. In the application under the delay perception model, sensor nodes form a fan-shaped perception area on a twodimensional plane. The monitoring task in the delayed coverage does not require high timeliness of the monitored data, and a possible sensing area can be formed by the rotation of the node. The sensor nodes are uniformly and randomly distributed in the monitoring area, satisfying the Poisson point distribution process. And the ability of the sensor to monitor the target depends on the target's understanding of the network. In the case of detection coverage, the target does not know the location of the node and randomly selects a path as a traversal path. The probability of its path being sensed depends on the node's sensing direction and node location. When only considering the position of the node, only the neighboring nodes of the path may monitor the path at this time. The probability of the path being monitored is the same as that of the traditional sensor network, which can be expressed by formula (3). When considering the node's perception of the direction factor, as shown in Figure 5, the node can only perceive the path along the path from OM to ON. Assuming that the angle of view offset of the node is represented by $a$, the angle AOP is represented by $O$, and the angle of rotation of the node in a unit time is represented by $b$, the angle range of the node's perception direction that may perceive the path is

$$
\text { Angle }=a+O+b
$$

Among them, $O$ can be expressed as

$$
O=2 \times \arccos \left(\frac{d}{r}\right)
$$

Among them, $d$ is the distance from the node to the path, and $r$ is the perception radius of the node. The path can be monitored only when the node is within the angle range of the node's perception direction. Due to the randomness of the node's direction, the probability that a single node can perceive the path is 


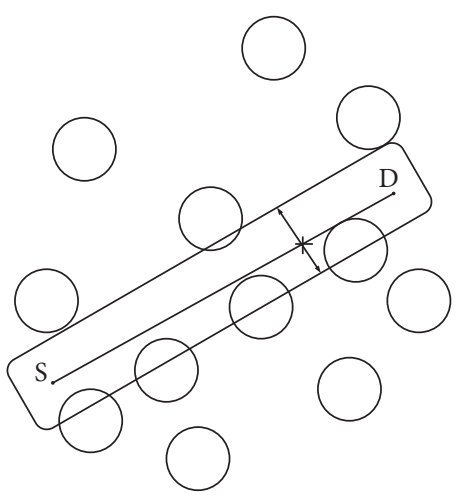

FIgURE 4: The straight path strategy.

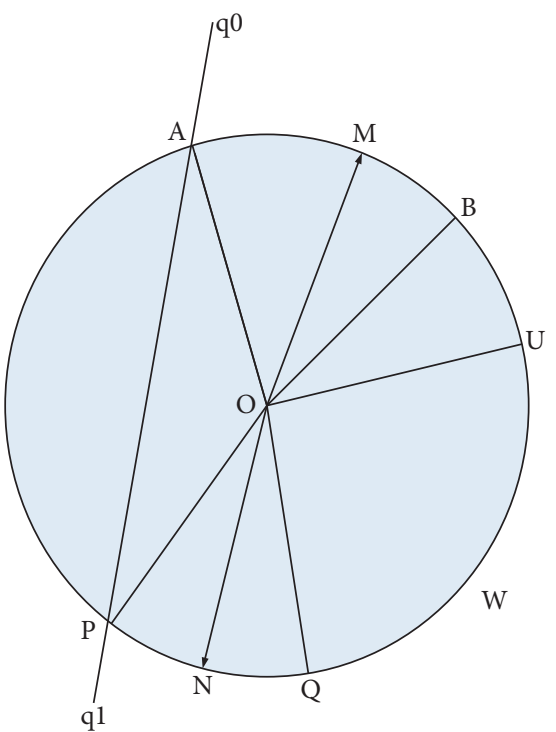

Figure 5: Delayed sensing model.

$$
Q_{0}=\frac{a+O+b}{2 \pi}
$$

Therefore, the probability that a single node cannot perceive the path is

$$
Q_{n o}=1-q_{0} \text {. }
$$

Since the nodes are randomly and evenly distributed in the monitoring area, it is assumed that the target randomly selects a path to traverse the monitoring area, and the number of adjacent nodes in the tubular area formed by the path is $n$. Then, the probability that all neighboring nodes cannot detect the path due to the sensing direction is

$$
Q_{N}=\left(1-Q_{0}\right)^{n} \text {. }
$$

Therefore, the probability that at least one node can monitor the path due to the problem of the node's perception of the direction factor is

$$
Q_{a}=1-Q_{N}
$$

Considering the location and sensing the direction of the sensor node, the probability that the target will be detected by at least one node while traveling along the selected path is

$$
Q=Q_{d} \times Q_{a}
$$

In the above analysis of the path monitoring capability, the nodes are randomly and evenly distributed, so it can be predicted that the number of nodes in the tube area meets

$$
n=\lambda \times\left\|Z_{q}\right\| \text {. }
$$

When the number of nodes in the monitoring area reaches a certain level, the distance between neighboring nodes and the path can be approximated by the average value, which is recorded as half of the sensing radius; then,

$$
O=2 \times \arccos \left(\frac{1}{2}\right)
$$

2.3. Judgment Rules for Node Rotation Direction. The rotation direction of the node is determined by the position of the centroid of the overlapping area. The centroid of the overlapping area describes the distribution of the overlapping area in the sensing area (this overlapping area refers to the union of all adjacent points and the overlapping area of the current node). When the centroid of the overlapping area is located in the right half of the sensing area of the node, it means that more overlapping areas are concentrated in the right half of the sensing area, and the node needs to rotate counterclockwise to reduce the overlap; conversely, when it is in the left half of the area, the node should rotate clockwise; and if it happens to be in the sensing direction, it means that the overlapping area is equal on the left and right sides, and the node randomly chooses the direction of rotation to reduce the overlap [18]. According to the above thought, the judgment rule of the rotation direction of the node can be expressed by the magnitude relationship between the direction angle $\phi_{1}$ and the deflection angle $\beta_{1}$ of the center of mass. If the current node does not have an overlapping area, and the node does not need to adjust the sensing direction, there is no need for the deflection angle of the centroid. When the node is partially overlapped, the deflection angle of the centroid must be required to determine the rotation direction of the node. Therefore, the position coordinate $\operatorname{Cen}_{i}(x 1, y 1)$ of the centroid of the overlapping area must be calculated first.

The abscissa of the centroid of the overlapping area of node $S_{i}$ is as follows:

$$
x_{\mathrm{Cen}_{i}}=\frac{\sum_{p=1}^{Z} \sum_{q=1}^{X} C g_{i p q} \times x_{i p q}}{\sum_{p=1}^{Z} \sum_{q=1}^{X} C g_{i p q}} .
$$

The ordinate of the centroid of the overlapping area of node $S_{i}$ is as follows:

$$
y_{\mathrm{Cen}_{i}}=\frac{\sum_{p=1}^{Z} \sum_{q=1}^{X} C g_{i p q} \times y_{i p q}}{\sum_{p=1}^{Z} \sum_{q=1}^{X} C g_{i p q}} .
$$

In formulas (14) and (15), $x_{i p q}$ and $y_{i p q}$ are the abscissa and ordinate of the $p$-th row and $q$-th column of node $S_{i}$, respectively. After calculating the centroid coordinates of the overlapping area by formulas (14) and (15), the Euclidean 
distance between it and the node coordinates can be calculated using formula (16):

$$
d_{i}=\sqrt{\left(x_{\mathrm{Cen}_{i}}-x_{P i}\right)^{2}+\left(y_{\mathrm{Cen}_{i}}-y_{P i}\right)^{2}} \text {. }
$$

Among them, $x_{i p q}$ and $y_{i p q}$ are the abscissa and ordinate of node $S_{i}$, respectively. Taking into account the rotation characteristics of the sensor node, we calculate the deflection angle of the center of mass in two cases according to the ordinate of the node and determine the rotation direction of the node based on this.

When $y 1_{\mathrm{Cen}_{i}}<y_{P i}$, the deflection angle of the center of mass is

$$
\beta_{i}=2 \pi-\left|\arccos \left(\frac{x_{\mathrm{Cen}_{i}}-x_{P_{i}}}{d_{i}}\right)\right| .
$$

When $y 1_{\mathrm{Cen}_{i}} \geq y_{P i}$, the deflection angle of the center of mass is

$$
\beta_{i}=\left|\arccos \left(\frac{x_{\mathrm{Cen}_{i}}-x_{P_{i}}}{d_{i}}\right)\right| .
$$

After calculating the deflection angle of the center of mass according to the above formula, the rotation direction of the node can be judged according to the relationship between the direction angle and the deflection angle of the center of mass. Figure 6 shows the agent mode of the virtual classroom. The specific judgment rules are divided into the following two situations.

If $0 \leq \phi_{i} \leq 2 \pi-\alpha$, so

$$
\begin{cases}\text { Clockwise, } & \text { when } \phi_{i}<\beta_{i}, \\ \text { Random rotation, } & \text { when } \phi_{i}=\beta_{i}, \\ \text { Turn counterclockwise, } & \text { otherwise. }\end{cases}
$$

If $2 \pi-\alpha<\phi_{i}=2 \pi$, so

$$
\begin{cases}\text { Turn counterclockwise, } & \text { when } \phi_{i}<\beta_{i}, \\ \text { Random rotation, } & \text { when } \phi_{i}=\beta_{i}, \\ \text { Clockwise, } & \text { otherwise. }\end{cases}
$$

Different from the traditional virtual force algorithm, OSRCEA puts forward the concept of overlapping perception ratio, which maps the coverage relationship between nodes to the ratio of overlapping area to perception area, and quantifies the rotation angle of the node through scalar operation, which greatly reduces the complexity of the algorithm. At the same time, the grid method is used to calculate the area of the overlapped area of the node to ensure the acquisition of the optimal angle of the node and finally to optimize the network coverage performance.

\section{Application of Campus Sports Information Teaching}

Through the reading and research of many literature studies on physical education at home and abroad, the existing theoretical research on teaching and physical education is

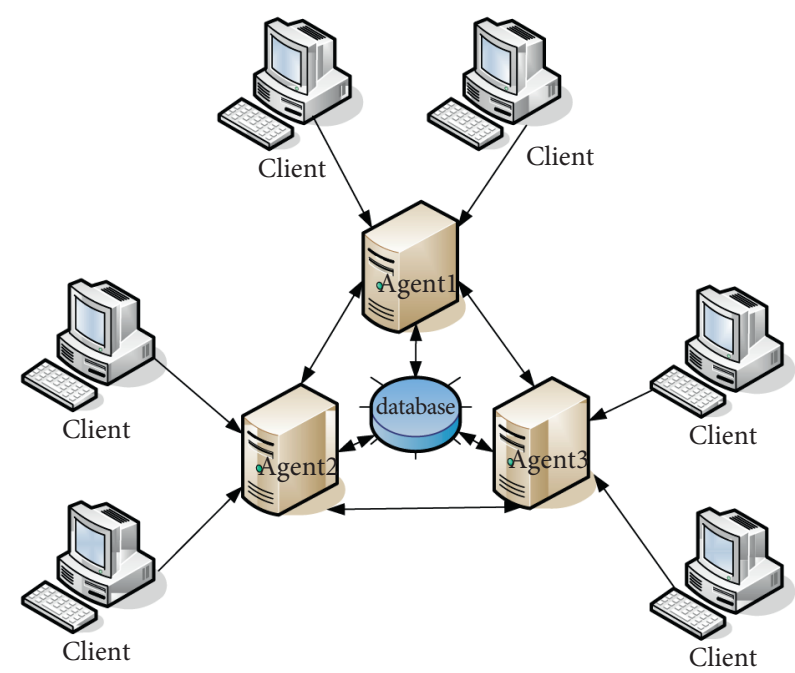

Figure 6: Virtual classroom agent mode.

summarized, and through horizontal comparison, problems are found and research problems are summarized in this paper. The research content is divided according to the relevant indicators, listed on the questionnaire; we select those users who meet the requirements and find out the problems in it through the questionnaire survey [3]. Generally, we include the following aspects:

Questionnaire 1, the questionnaire for teachers is mainly used for the key points and difficulties of sports technology teaching.

Questionnaire 2, the questionnaire for students mainly understands the difficulties of sports learning.

Questionnaire 3, the investigation of the application effect of informatization teaching in physical education is mainly used to understand the learning effect of the course.

Questionnaire 4, to understand the students' feelings and interest in physical education courses after using informatized teaching.

3.1. The Role of Informatization Teaching in the Long Jump Project at the Junior High School Stage

(1) The long jump event is a process assessment item for the second grade of junior high school. The assessment standard is shown in Table 1 . It is difficult to teach, requires high movement skills, and is difficult to learn. The long jump project is also a project for the development of whole-body coordination and explosive power. In the assessment, most students are difficult to meet the assessment standards [19], and both teachers and students have certain challenges.

(2) Purpose of the teaching experiment

Through the comparative teaching method, the students in the two classes were taught by the 
TABLE 1: Long jump achievement standards for the second grade of junior high school.

\begin{tabular}{lcc}
\hline \multirow{2}{*}{ Score (fraction) } & \multicolumn{2}{c}{ Unit (meter) } \\
\hline 100 & Boy (meter) & Girl (meter) \\
98 & 4.35 & 3.49 \\
96 & 4.30 & 3.46 \\
94 & 4.24 & 3.42 \\
92 & 4.21 & 3.39 \\
90 & 4.18 & 3.37 \\
86 & 4.13 & 3.35 \\
84 & 4.03 & 3.28 \\
80 & 3.92 & 3.21 \\
78 & 3.87 & 3.18 \\
75 & 3.81 & 3.14 \\
73 & 3.70 & 3.07 \\
69 & 3.59 & 3.00 \\
67 & 3.49 & 2.93 \\
64 & 3.45 & 2.88 \\
60 & 3.38 & 2.80 \\
\hline
\end{tabular}

traditional teaching mode and the combination of assisted teaching and traditional teaching, through the comparative analysis of the comparative results, to explore the advantages of information-assisted teaching compared with the traditional teaching mode.

(3) Teaching experiment method

In this study, two classes in the second grade of a school affiliated with a university were selected as the research objects. A total of 60 students from the two classes participated in this teaching experiment research. Among them, there are 17 boys and 13 girls in the experimental group and 19 boys and 11 girls in the control group, all of them belong to ordinary classes, and the students in the class have average grades. There are no students with special skills in sports. The students are active and well disciplined in class. Comparative studies can be carried out; the ratio of male to female students in the two classes is not much different. In the teaching experiment, a total of 6 class hours of research was conducted in May-June. The teaching site and content of the two classes were the same, and the teaching location was in the sandpit on the east side of the playground. Among them, the control group uses conventional teaching methods to teach, and the experimental group adds a large amount of information teaching on the basis of conventional teachings, such as pictures, videos, and technical actions recorded by students during class. We made a comparative study of the teaching progress, results, quality, efficiency, and other aspects of the two classes.

Class 1: in the classroom, the teacher makes a good PPT, lets the students watch the technical movements of the long jump and related videos, stimulates the students' interest in learning, guides the students to ask questions, and analyzes them.
Class 2: in the playground, the teacher teaches the technical essentials of the run-up, learns the rhythm of the run-up, and focuses on practicing the rhythm of the board. The professor uses the big screen to remotely play the excellent video actions of the runup on the board. Students record their technical actions during the practice. The focus of this lesson is to use the video playback on the big screen to give students an intuitive display.

Class 3: in the playground, the teacher teaches the technical movements of take-off, three-step take-off, and five-step take-off, focusing on practicing the continuity of the active swing of the swing leg and the approach and take-off. During the class, the teacher uses the big screen to remotely broadcast the technical movements of the jump and selectively plays the technical video of the students in the previous class, which takes about 5-10 minutes. When students are practicing, they can selectively record and play wrong actions of students and let students conduct self-examination.

Class 4: the teacher teaches the technical movements of the abdomen and knee bend, first completes on the mat, and experiences the technical movements of landing kicking forward. In class, the technical movement of kicking and stretching is played to students in slow motion. When practicing the technical movements of the abdomen and knee bend, students can selectively play mistakes that students make easily, so that students can watch them intuitively and make corrections.

Class 5: the teacher allows students to experience complete technical movements. Teachers play videos of complete technical actions to students in class so that students can experience the continuity of actions and play them in real time. Students watch technical actions and correct wrong actions.

In class 6, the evaluation of the long jump is carried out. Three teachers are used to evaluate the technical evaluation, and the important and difficult points of the technical actions are scored and evaluated, and the students are recorded and played in real time. Questionnaires are issued to students after class, mainly to understand the investigation of students' interest in learning long jump, the influence of information teaching on learning interest, the influence on teaching ability, the influence on classroom atmosphere, and so on.

Table 1 shows the long jump scoring standards for boys and girls, and Figure 7 shows the comparison of scoring standards.

3.2. Difficulties in Long Jump Teaching and Learning. Analyzing the teaching difficulties of traditional teaching is the basis of using informatization teaching. The use of informatization teaching methods to analyze the teaching difficulties in traditional teaching will help to make better use of informatization to achieve the purpose of optimizing 

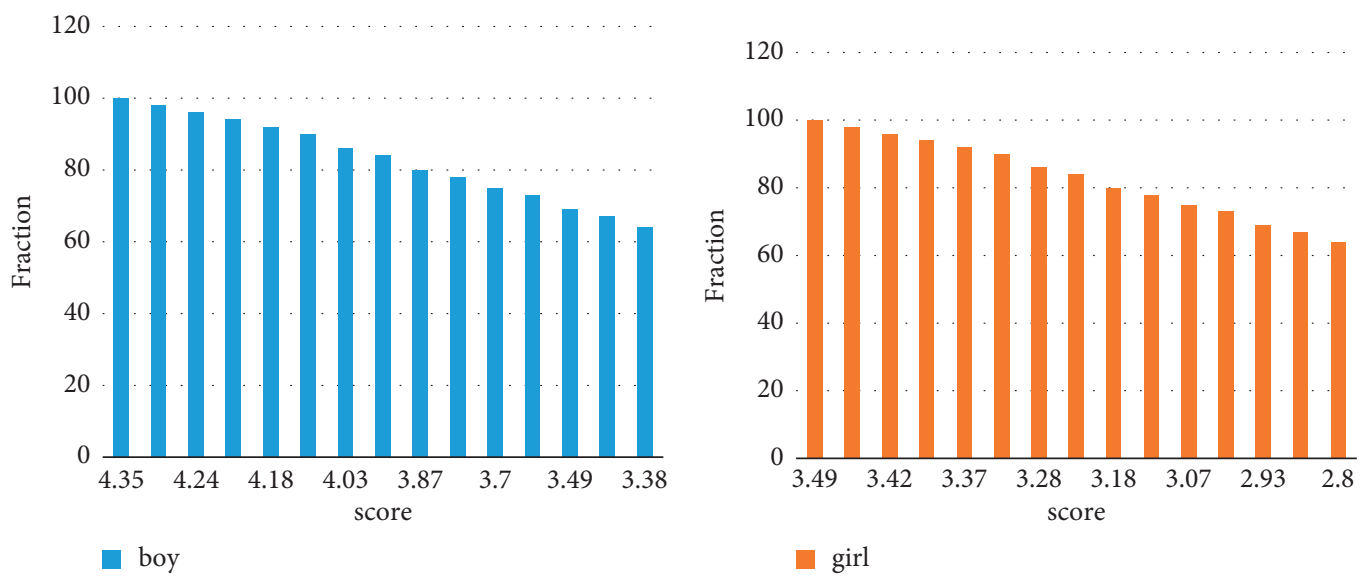

Figure 7: Boys and girls score sheet.

teaching. According to teacher surveys, teachers believe that in the learning process, the decomposition of long jump teaching is more difficult to teach running, taking off, flying, and landing. For the difficulty of learning, the results of the students' survey are shown in Table 2.

\subsection{Comparison of Teaching Effect between Informationized} Teaching and Conventional Teaching. After the teaching experiment, Table 3 lists the relevant data collected by the questionnaire method, which mainly includes the students' attitude data to this research-centric teaching method.

From the above data, it is clear that the students in the experimental group agree with this teaching method and think that it can help to stimulate interest in sport and improve results. In addition, the data from the answers to the four questions $3,4,11$, and 12 in the questionnaire show that this teaching method has influenced the students in the experimental group and encouraged them to actively try to apply this teaching model to other subjects. The data from the answers to the four questions six to nine show that effective implementation of the teaching method helps to improve the relationships between teachers, pupils, and classmates and has some effect on promoting pupils' teamwork skills.

\subsection{Comparison of the Results of the Technical Assessment of} the Long Jump between the Experimental and Control Groups. The main content of the long jump technical evaluation is the evaluation of the students' performance of the technical actions involved in the long jump, which is undoubtedly an important teaching content. In lessons lasting 12 school hours, teachers use the last school hour to assess pupils' long jump skills. The assessment consists of two parts: the assessment of theoretical knowledge and the performance of basic actions. The result of each part is weighted by 50 points, and the total score is used as an assessment of the pupil's technical evaluation. Table 4 provides information on the results of the experimental and control group performance tests for the long jump project and the associated technical actions; the details are as follows.
3.5. Two Groups of Students' Learning Interest. The statistics in Table 5 show that after the experimental training almost half of the students were interested in learning the long jump at all. It can be seen that the experimental group was more interested in learning the long jump than the control group. The long jump has quite high demands in terms of technical movements. Not only do students need to learn actively under the guidance of their teachers, but more importantly, they need to have a spirit of active learning. Only by effectively combining both sides can their learning be effectively enhanced [20].

The level of question analysis skills can determine how much insight a student has into a problem; that is, it can reflect whether the student has a good ability to analyze the substance of something based on its appearance. The core of problem analysis skills is to analyze the problem, then identify the cause of the problem, and eventually find a solution to the problem. Figure 8 shows a two-group comparison of students' ability to both identify and deal with problems. In the long jump class, both the control and experimental groups had to have the ability to analyze problems, but after exercises with different teaching methods, Table 6 combines the final evaluation of the students by the teachers. Figure 9 shows the comparison of the number of students interested in the different pedagogical methods.

\section{Discussion}

The control group students using the traditional teaching model are basically passive learning subjects, lacking the corresponding interaction and learning experience, so the students' learning interest is difficult to cultivate and lack learning initiative [21]. The students in the experimental group watched their own technical action videos through teaching and learning methods to make corrections. In this way, it is more able to choose with one's own learning path and degree, which helps to enhance the student's subjective initiative in learning. Based on this, it can further cultivate students' interest in learning. The teacher's guidance and help can also effectively stimulate students' interest in learning, thereby effectively improving teaching efficiency and effectiveness [22]. 
TABLE 2: Difficulties in learning long jump.

\begin{tabular}{lccccc}
\hline Teaching content & Theoretical study & Approach & Take-off & Vacate & Landing \\
\hline Number of people & 6 & 22 & 34 & 45 & 42 \\
Percentage & 10.11 & 38.22 & 55.00 & 76.65 & 68.35 \\
\hline
\end{tabular}

TABLE 3: Investigation of the students' learning attitudes in the experimental group after the experiment $n=30$.

\begin{tabular}{|c|c|c|c|c|c|}
\hline Label & Questionnaire content & A (\%) & $\mathrm{B}(\%)$ & $\mathrm{C}(\%)$ & $\mathrm{D}(\%)$ \\
\hline 1 & The degree of fondness for informatization teaching & 51.4 & 41.7 & 4.2 & 0 \\
\hline 2 & The influence of informatization teaching on learning interest & 62.5 & 24 & 12.5 & 0 \\
\hline 3 & Attitudes to use informatization teaching for other teachings & 45.8 & 41.7 & 12.5 & 0 \\
\hline 4 & The influence of informatization teaching on teaching ability & 25 & 63.5 & 12.5 & 0 \\
\hline 5 & The influence of informatization teaching on classroom atmosphere & 37.5 & 45.7 & 16.7 & 0 \\
\hline 6 & The influence of informatization teaching on classroom student relationship & 37.5 & 41.7 & 20.8 & 0 \\
\hline 7 & The influence of informatization teaching on the relationship between teachers and students in class & 83.2 & 12.4 & 4.2 & 0 \\
\hline 8 & Are you willing to help students with practice & 50 & 41.7 & 4.2 & 4.2 \\
\hline 9 & Teaching effect of classroom teaching and demonstration & 20.5 & 41.7 & 29.2 & 8.3 \\
\hline 10 & Evaluation attitude towards teachers and classmates & 34.3 & 57.3 & 8.2 & 0 \\
\hline 11 & The influence of informatization teaching on future teaching practice & 70.8 & 25 & 4.2 & 0 \\
\hline 12 & The influence of informatization teaching on the ability of data collection and Writing & 37.5 & 51 & 12.5 & 0 \\
\hline 13 & The influence of informatization teaching on mastering technical skills & 41.7 & 41.7 & 16.5 & 0 \\
\hline 14 & Attitudes towards evaluation methods of teaching & 53.2 & 41.5 & 4.2 & 0 \\
\hline 15 & After class study and actual exercises & 41.7 & 33.3 & 20.7 & 0 \\
\hline
\end{tabular}

TABLE 4: Comparative analysis of the long jump technical evaluation scores of the experimental group and the control group $n=60$.

\begin{tabular}{lcccc}
\hline Grade type & Group & Number of people & Average & $T$ \\
\hline Initial evaluation score & Test group & 30 & 61.2 & \\
\multirow{2}{*}{ Evaluation results } & Control group & 30 & 61.3 & \\
& Test group & 30 & 84.52 & -3.21 \\
& Control group & 30 & 73.42 & $<0.05$ \\
\hline
\end{tabular}

TABLE 5: Comparative analysis of long jump learning interest of students in the experimental group and the control group $n=60$.

\begin{tabular}{lccccc}
\hline & & Very interested & Interested & Generally & Not interested \\
\hline \multirow{2}{*}{ Test group } & $n$ & 24 & 20 & 16 & 0 \\
& $\%$ & $40 \%$ & $33.3 \%$ & $26.6 \%$ & 0 \\
Control group & $n$ & 20 & 14 & 26 & 0 \\
& $\%$ & $33.3 \%$ & 23.3 & $43.3 \%$ & 0 \\
\hline
\end{tabular}

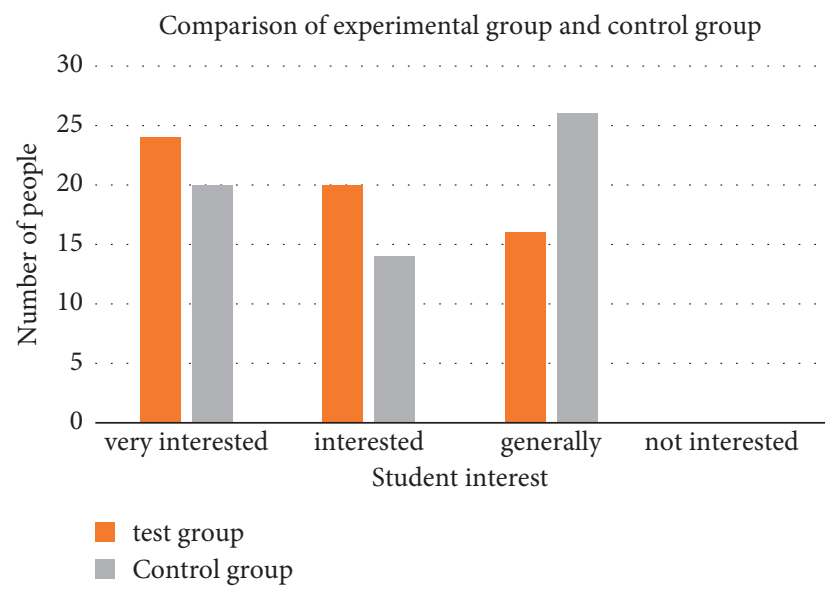

Figure 8: Problem analysis comparison. 
TABLE 6: Comparison of long jump analysis and problem-solving ability of students in the experimental group and the control group $n=60$.

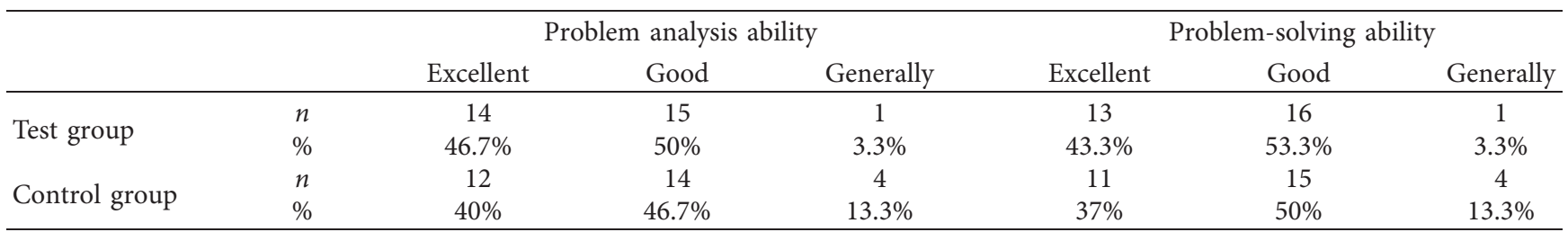
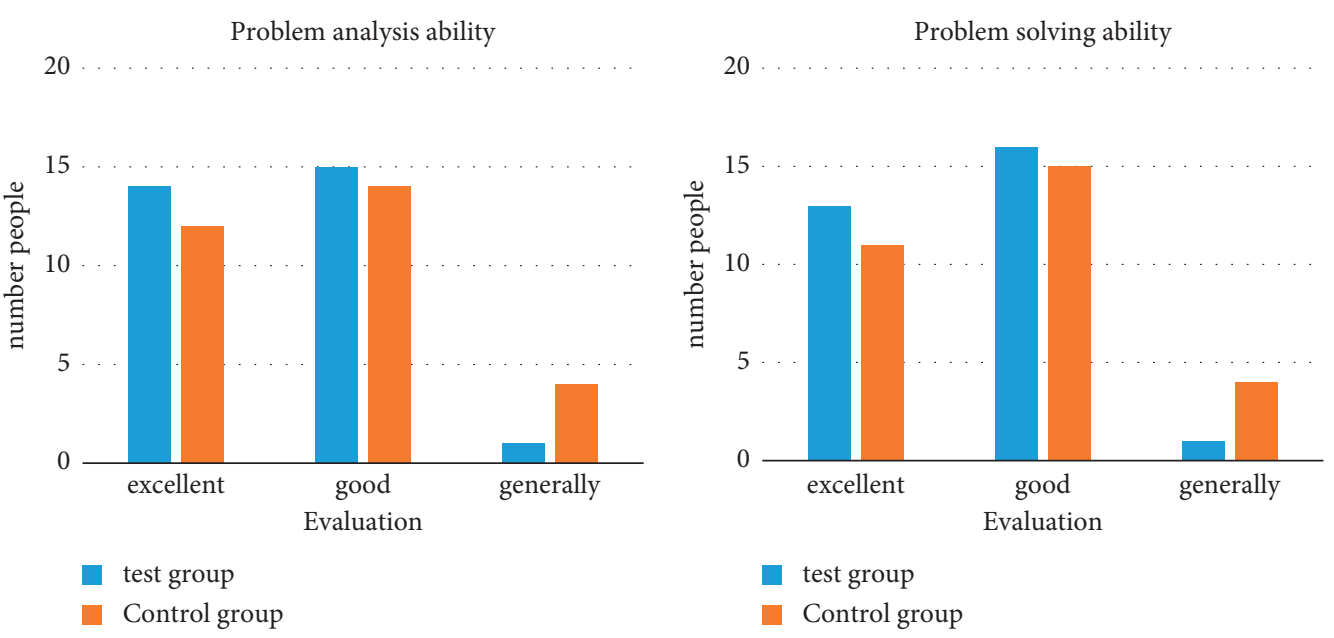

Figure 9: Comparison of experimental group and control group.

TABLE 7: Comparative analysis of long jump teaching efficiency of experimental group and control group students.

\begin{tabular}{lccc}
\hline & Teaching time (frequency) & Evaluation results (minute) & Teaching efficiency (\%) \\
\hline Test group & 12 & 93.7 & 94.4 \\
Control group & 15 & 80.5 & 81.4 \\
$P$ & $<0.06$ & $<0.06$ & $<0.06$ \\
\hline
\end{tabular}

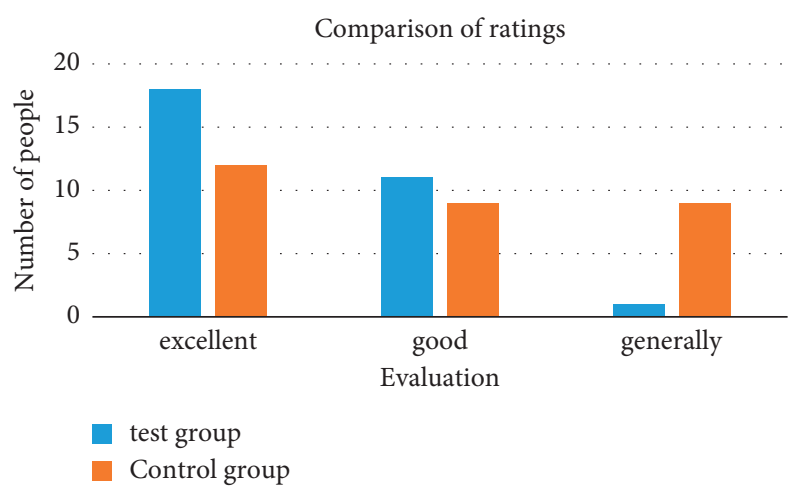

Figure 10: Take-off technique master comparison chart.

According to Table 7, the use of teaching can intuitively and vividly show students the theory and technical movements of the long jump. And through detailed display, decomposition of movement analysis and other methods let students fully master the technical essentials, so that students have more time to practice and improve students' long jump skills [23].

In this study, two groups of students were taught in different ways. After the completion of the teaching, all the students who participated in the experiment were tested in a unified long jump technique. After the test, the two groups of students' mastery of long jump techniques were statistically analyzed, as shown in Figure 10 and Figure 11. It can be seen that the excellent students in the take-off technique and approach technique of the experimental group students accounted for more than $60 \%$, and the students with average technique only accounted for $3 \%$ with only one classmate. Among the students in the control group, excellent students accounted for $40 \%$, while the average technical evaluation students reached $30 \%$. It can be seen that the students in the control group did not have a thorough understanding of the 


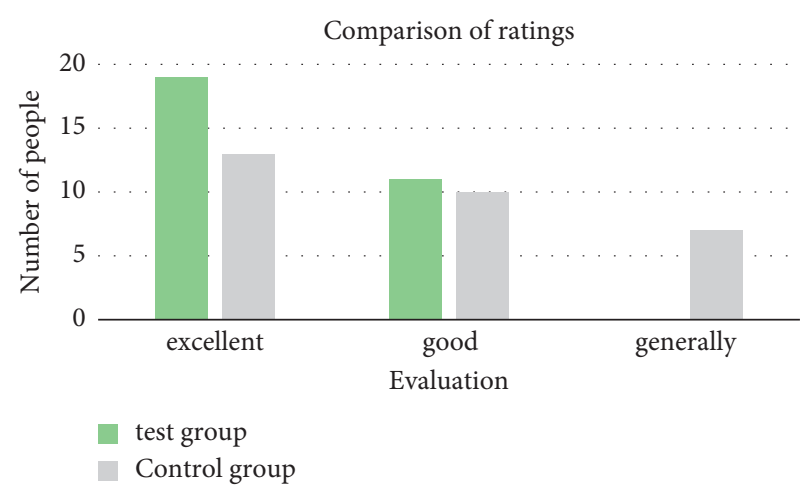

FIGURE 11: Comparison chart of approach technique mastery.

technical movements of the long jump. In this study, the experimental group was compared with the control group students, the students can better master the technical movements of the long jump, and the mastery is much higher than the control group [24].

\section{Conclusion}

Technology centralizes the management of various pieces of information, whether it is text, pictures, animation, or video; it can be well interpreted, so it has good performance capabilities, allowing students to devote more enthusiasm to learning. The survey results show that $73.3 \%$ of the students who use teaching like long jump and only $56.6 \%$ of the students in the control group who use conventional teaching are interested in learning long jump. Learning interest can improve students' performance to a large extent, and it has a great effect on people's work and life. Through the experimental results, it is found that once the students are interested in learning, they will put more enthusiasm, their attention will be more concentrated, and the learning effect will be greatly improved. However, informatization teaching is also inadequate; the equipment involved in informatization technology is generally operated indoors. However, for physical education, training and demonstration are required outdoors in many cases, and the learning of some skills is also the case. Therefore, as far as physical education is concerned, information-based teaching is generally done indoors, and there is less outdoor teaching, which is more restricted by the venue.

\section{Data Availability}

No data were used to support this study.

\section{Conflicts of Interest}

The authors declare that they have no conflicts of interest.

\section{Acknowledgments}

This work was supported by the Ministry of Education of the People's Republic of China: Based on the Information Technology of Physical Education and Health Management; online sports classes are built on campus (no. 202002012031).

\section{References}

[1] M. Fadi and Al-Turjman, "Information-centric sensor networks for cognitive IoT: an overview," Annals of Telecommunications, vol. 72, no. 1-2, pp. 3-18, 2016.

[2] W. Gong, L. Tong, W. Huang, and S. Wang, "The optimization of intelligent long-distance multimedia sports teaching system for IOT," Cognitive Systems Research, vol. 52, pp. 678-684, 2018.

[3] R. Borawake-Satao and R. Prasad, "Comprehensive survey on effect of mobility over routing issues in wireless multimedia sensor networks," International Journal of Pervasive Computing and Communications, vol. 12, no. 4, pp. 447-465, 2016.

[4] C. Tang, Q. Tan, and Y. Han, "An energy harvesting aware routing algorithm for hierarchical clustering wireless sensor networks," KSII Transactions on Internet and Information Systems (TIIS), vol. 2, pp. 504-521, 2016.

[5] L. Li, J. Wang, J. Wang, C. Zhang, L. Tian, and W. Zhu, "Construction and practice of multi-information teaching mode of inorganic chemistry," University Chemistry, vol. 34, no. 4, pp. 34-38, 2019.

[6] K. Sohn, Y. Y. Kim, and N. Saxena, "A new cross-layer QoSprovisioning architecture in wireless multimedia sensor networks," KSII Transactions on Internet \& Information Systems, vol. 10, no. 12, pp. 5286-5306, 2016.

[7] Y. Noh, U. Lee, S. Lee et al., "HydroCast: pressure routing for underwater sensor networks," IEEE Transactions on Vehicular Technology, vol. 65, no. 1, pp. 333-347, 2016.

[8] R. W. L. Coutinho, A. Boukerche, L. F. M. Vieira, and A. A. F. Loureiro, "Geographic and opportunistic routing for underwater sensor networks," IEEE Transactions on Computers, vol. 65, no. 2, pp. 548-561, 2016.

[9] I. Butun, P. Osterberg, and H. Song, "Security of the Internet of things: vulnerabilities, attacks, and countermeasures," IEEE Communications Surveys \& Tutorials, vol. 22, no. 1, pp. 616-644, 2020.

[10] J. Ren, Y. Zhang, K. Zhang, A. Liu, J. Chen, and X. S. Shen, "Lifetime and energy hole evolution analysis in data-gathering wireless sensor networks," IEEE Transactions on Industrial Informatics, vol. 12, no. 2, pp. 788-800, 2016.

[11] C. Benzaid, K. Lounis, A. Al-Nemrat, N. Badache, and M. Alazab, "Fast authentication in wireless sensor networks," Future Generation Computer Systems, vol. 55, pp. 362-375, 2016.

[12] J. Jiang, G. Han, and F. Wang, "An efficient distributed trust model for wireless sensor networks," IEEE Transactions on Parallel and Distributed Systems, vol. 26, no. 5, pp. 1228-1237, 2016.

[13] V. Annepu and A. Rajesh, "An unmanned aerial vehicle-aided node localization using an efficient multilayer perceptron neural network in wireless sensor networks," Neural Computing \& Applications, vol. 32, no. 15, pp. 11651-11663, 2020.

[14] H. Zhang, X. Hong, J. Cheng, A. Nallanathan, and V. C. M. Leung, "Secure resource allocation for OFDMA twoway relay wireless sensor networks without and with cooperative jamming," IEEE Transactions on Industrial Informatics, vol. 12, no. 5, pp. 1714-1725, 2017.

[15] M. Dong, K. Ota, and A. Liu, "RMER: reliable and energyefficient data collection for large-scale wireless sensor networks," IEEE Internet of Things Journal, vol. 3, no. 4, pp. 511-519, 2017.

[16] G. Han, J. Jiang, and C. Zhang, "A survey on mobile anchor node assisted localization in wireless sensor networks," IEEE Communications Surveys \& Tutorials, vol. 18, no. 3, pp. 2220-2243, 2017. 
[17] C. Zhu, H. Wang, X. Liu, L. Shu, L. T. Yang, and V. C. M. Leung, "A novel sensory data processing framework to integrate sensor networks with mobile cloud," IEEE Systems Journal, vol. 10, no. 3, pp. 1125-1136, 2016.

[18] Z. Fei, B. Li, S. Yang, C. Xing, H. Chen, and L. Hanzo, "A survey of multi-objective optimization in wireless sensor networks: metrics, algorithms, and open problems," IEEE Communications Surveys \& Tutorials, vol. 19, no. 1, pp. 550-586, 2017.

[19] W. Zhuo, R. Lu, and D. Chen, “An experience information teaching-learning-based optimization for global optimization," IEEE Transactions on Systems Man \& Cybernetics Systems, vol. 46, no. 9, pp. 1202-1214, 2016.

[20] H. Osouli Tabrizi and F. Al-Turjman, "AI for dynamic packet size optimization of batteryless IoT nodes: a case study for wireless body area sensor networks," Neural Computing \& Applications, vol. 32, no. 20, pp. 16167-16178, 2020.

[21] T.-H. Jiang, S.-L. Chen, and J. K. C. Chen, "Examining the role of behavioral intention on multimedia teaching materials using FSQCA," Journal of Business Research, vol. 69, no. 6, pp. 2252-2258, 2016.

[22] B. Pitchaimanickam and G. Murugaboopathi, "A hybrid firefly algorithm with particle swarm optimization for energy efficient optimal cluster head selection in wireless sensor networks," Neural Computing \& Applications, vol. 32, no. 12, pp. 7709-7723, 2020.

[23] L. Cardenas Cantos, J. Lopez Izquierdo, and E. Cardenas Cantos, "Interactive multimedia application for teaching and learning in Analytical Geometry," IEEE Latin America Transactions, vol. 14, no. 7, pp. 3461-3466, 2016.

[24] P. Liu, H. Cui, Y. Cao, X. Hou, and L. Zou, "A method of multimedia teaching evaluation based on fuzzy linguistic concept lattice," Multimedia Tools and Applications, vol. 78, no. 21, pp. 30975-31001, 2019. 